\title{
Modification of the Nonanalytic Equation of State for the Limit of Low Densities*
}

\author{
Robert D. Goodwin \\ Institute for Basic Standards, National Bureau of Standards, Boulder, Colorado 80302
}

April 4, 1978

\begin{abstract}
As described in previous reports, this equation is constrained to the liquid-vapor coexistence boundary. Integration of the "thermodynamic equation of state" along isotherms involves a term for the compressibility factor of saturated vapor in the limit of low densities, which in previous work was not adequately defined. The present, brief report assumes familiarity with previous work, and presents a solution for the problem, which is to describe saturated-vapor densities in terms of the compressibility factor for saturated vapor, utilizing a given, vaporpressure equation.
\end{abstract}

Key words: Equation of state; ethylene; internal energy; saturated vapor densities; thermodynamic properties; vapor pressures.

\section{Symbols and Units}

Subscripts $c$ and $t$ refer to critical and liquid triple points.

Subscript $\sigma$ refers to liquid-vapor coexistence.

$d \quad$ density, $\mathrm{mol} / \mathrm{L}$

$E \quad$ the internal energy, $\mathrm{J} / \mathrm{mol}$

$J \quad$ the joule, $1 N-m$

$L \quad$ the liter, $10^{-3} \mathrm{~m}^{3}$

mol $\quad 28.054$ grams of ethylene $\left(C^{12}\right.$ scale $)$

$P \quad$ pressure in bars, 1 bar $\equiv 10^{5} \mathrm{~N} / \mathrm{m}^{2},(1 \mathrm{~atm}=$ 1.01325 bar)

$P_{\sigma}(T) \quad$ vapor pressure, bar

$P_{\sigma}(\rho) \quad P_{\sigma}\left[T_{\sigma}(\rho)\right]$ for equation (1)

$R \quad$ the gas constant, $8.31434(\mathrm{~J} / \mathrm{mol}) / K, 0.0831434$

(bar- $L / \mathrm{mol}) / K$

R* $\quad(0.0831434) \cdot d_{\mathrm{c}}$, bar $/ K$

$\rho \quad d / d_{\mathrm{c}}$, reduced density

$T \quad$ temperature, $K$

$T_{\sigma}(\rho) \quad$ liquid-vapor coexistence temperature

\section{Introduction}

Adaptations of this type of equation of state have been described in numerous reports $[1-8],{ }^{1}$ which must serve the interested reader as background, to avoid repetition here. The form of this isochoric equation is

$$
P-P_{\sigma}(\rho)=\rho R^{*} \cdot\left[T-T_{\sigma}(\rho)\right]+\rho^{2} R^{*} T_{c} \cdot F(\rho, T),
$$

in which, for any given density (isochore), the coexistence

\footnotetext{
* This work was supported by the American Gas Association, Inc., 1515 Wilson Boulevard, Arlington, VA 22209.

${ }^{1}$ Figures in brackets refer to literature references at the end of this paper.
}

temperature, $T_{\sigma}(\rho)$, is obtained by iteration from equations for the orthobaric densities. This is used to obtain the vapor pressure, $P_{\sigma}\left[T_{\sigma}(\rho)\right]$, as a function of density. The function $F(\rho, T)$ contains terms roughly analogous to $T$ and $1 / T^{2}$ of the well-known, Beattie-Bridgeman equation of state, each with a density-dependent coefficient. Symbols and units for this report are given in a list. Fixed-points, used for some examples on ethylene, are given in table 1.

TABLE 1. Fixed-Point Values Used for Ethylene

\begin{tabular}{ccc}
\hline & Triple Point & Critical Point \\
\hline Temperature, K & 103.97 & 282.35 \\
Pressure, bar & $1.2095 \cdot 10^{-3}$ & 50.4234 \\
Density mol/L & & \\
Vapor & $1.3994 \cdot 10^{-4}$ & 7.60 \\
Liquid & 23.3590 & 7.60 \\
\hline
\end{tabular}

\section{The Internal Energy}

Placing eq (1) in the thermodynamic equation of state yields

$$
\Delta E=\int_{0}^{\rho}\left[\left(Z_{\sigma}(\rho)-1\right) \cdot R T_{\sigma}(\rho) / \rho+\ldots\right] \cdot d \rho,
$$

where $E$ is the internal energy.

Our problem is in the definition and value of

$$
Z_{\sigma}(\rho) \equiv P_{\sigma}(\rho) /\left[\rho \cdot R^{*} \cdot T_{\sigma}(\rho)\right]
$$

in the limit of low densities, because equations for the vapor pressures, $P_{\sigma}(T)$, and for the saturated vapor densities, $\rho_{\sigma}(T)$, generally are independent. From equations which we 
used for propane, for example [6], we find that at $\rho=0$, (hence $\left.T_{\rho}(\rho)=0\right)$, the value of $Z_{\sigma}(\rho)$ may range from 0 to $\infty$. Even if $Z_{\sigma}(\rho) \rightarrow 1$ at low densities, there remains the problem of loss of significant digits in the difference $\left[Z_{\sigma}(\rho)\right.$ $-1]$.

\section{Saturated Vapor Densities}

A solution for the problem of eq (2) is to describe saturated vapor densities in terms of their compressibility factors (utilizing the vapor-pressure equation) in such a way that the integrand in eq (2) will extrapolate to a reasonable value as $\rho \rightarrow 0$.

The following formulation utilizes temperature, $T$, as independent variable. If, for a given vapor density, however, the temperature has been found from this formulation by iteration, then $T \rightarrow T_{\sigma}(\rho)$, and the compressibility factor becomes a function of density, $Z_{\sigma}(T) \rightarrow Z_{\sigma}\left[T_{\sigma}(\rho)\right]$, as needed for eq (2), above.

Define the constant $A_{o} \equiv\left(Z_{c}-1\right)$, where $Z_{c} \equiv$ $P_{c} /\left(\rho_{c} \cdot R^{*} \cdot T_{c}\right)$ is value of the compressibility factor at the critical point, and the reduced variables,

$$
\Pi(T) \equiv P_{\sigma}(T) / P_{c}, x(T) \equiv T / T_{c}, u(T) \equiv 1-x,
$$

when the equation for saturated vapor densities,

$$
\rho_{\sigma}(T) \equiv P_{\sigma}(T) /\left[Z_{\sigma}(T) \cdot R^{*} \cdot T\right]
$$

is given by

$$
\begin{gathered}
Z_{\sigma}(T)=1+A_{o} \cdot \Pi \cdot x^{-2} \cdot f(x), \\
f(x) \equiv 1+A_{1} \cdot u^{\epsilon}+\sum_{i=2}^{5} A_{i} \cdot u^{i-1} .
\end{gathered}
$$

At $T=0, f(x)$ is finite, and $Z_{\sigma}(T)=1$, due to the exponential dependence of the vapor pressure, $P_{\sigma}(T) \sim$ $\exp (-1 / T)$

To give substance to these analytical results, we present the vapor-pressure equation for ethylene in appendix $A$, taken from an unpublished report. Figure 1 shows behavior of $f(x)$, eq (5), for which the constants are

$$
\begin{array}{rlrl}
\epsilon & =0.36 & A_{3}=0.6955385 \\
A_{1}=-0.8648073 & A_{4}=-3.064851 \\
A_{2}=0.8931234 & A_{5}=5.863340
\end{array}
$$

The rms relative deviation of the ethylene saturated vapor densities is 0.07 percent for a total of 29 points, including experimental data from 233 through $282 \mathrm{~K}$, and data from 120 through $220 \mathrm{~K}$ derived via the vapor-pressure and virial equations [7].

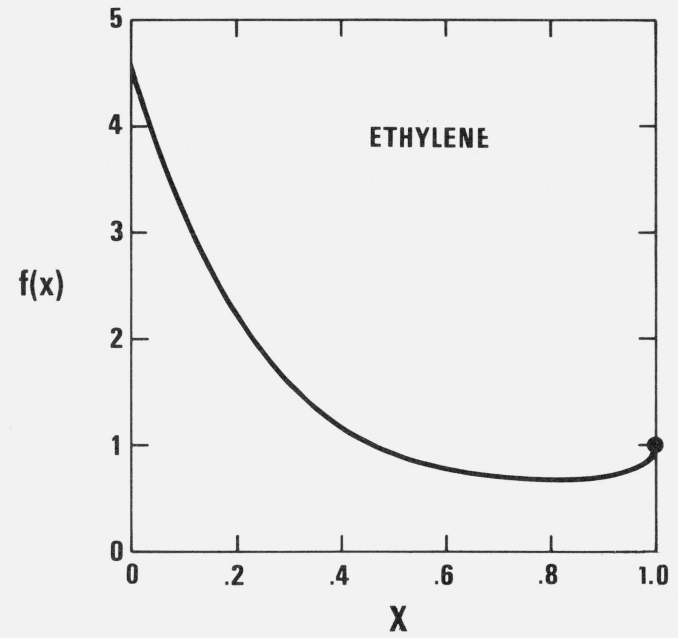

Figure 1. Behavior of $f(x)$, eq (5), for ethylene.

\section{Conclusions}

By use of eqs (3) and (4) for substitutions in eq (2) at densities $\rho \leq \rho_{c}$, one obtains

$$
\Delta E=\int_{0}^{\rho}\left[\left(Z_{c}-1\right) \cdot\left(Z_{\sigma}(\rho) / Z_{c}\right)\right.
$$

$$
\left.\cdot R T_{c} \cdot f(x)+\ldots\right] \cdot d \rho,
$$

in which the integrand is finite in the limit $\rho=0,\left(T_{\sigma}(\rho)=\right.$ $0)$.

Because the equation of state is nonanalytic, the integrations of eqs (2) or (2a) must be performed numerically. In published works $[4,5,6]$ we have not called upon the equation of state at densities so very low as to encounter the difficulty described and resolved in this report. At very low densities, the saturated vapor temperature diminishes roughly only as the logarithm of density, $1 / T \sim \ln (1 / \rho)$.

As it is improbable that we ever shall be privileged to compare thermodynamic properties from eq (2) with those from eq (2a), our objective in this report has been to describe the problem, and to give a solution for consideration in all future work.

\section{Appendix A. Ethylene Vapor-Pressure Equation}

For use in eq (3), above, our unpublished vapor-pressure equation for ethylene, from the triple- to the critical-point, for $P$ in bar, is

$\ln (P)=a+b \cdot u+c \cdot x+d \cdot x^{2}+$

$$
e \cdot x^{3}+f \cdot x \cdot(1-x)^{\epsilon},
$$


with arguments $x \equiv\left(T-T_{t}\right) /\left(T_{c}-T_{t}\right), u \equiv\left(1-T_{t} / T\right) /(1$ $\left.-T_{t} / T_{c}\right)$, where $\epsilon=1.30$, and

$$
\begin{array}{ll}
a=-6.7175716 & d=4.5536920 \\
b=13.8976040 & e=-1.1320564 \\
c=-6.6812125 & f=0.6236504
\end{array}
$$

For 38 data points from 104.9 through $282.3 \mathrm{~K}$, the rms of relative pressure deviations is 0.04 percent [7].

\section{References}

[1] Goodwin, R. D., Equation of state for thermodynamic properties of fluids, J. Res. Nat. Bur. Stand. (U.S.), 79A (Phys. and Chem.) No. 1, 71-79 (Jan.-Feb. 1975).

[2] Goodwin, R. D., An equation of state for thermodynamic properties of pure fluids, Fifth International CODATA Conference, Boulder, Colo., June 28, 1976. [International Council of Scientific Unions, 51 Boulevard de Montmorency, 75016, Paris, France.]

[3] Goodwin,R. D., On the nonanalytic equation of state for propane, Paper ID-1, Cryogenic Engineering Conference, Boulder, Colo., Aug. 2, 1977.

[4] Goodwin, R. D., The thermophysical properties of methane, from 90 to $500 \mathrm{~K}$ at pressures to $700 \mathrm{bar}$, Nat. Bur. Stand. (U.S.), Tech. Note 653, 280 pages (Apr. 1974).

[5] Goodwin, R. D., Roder, H. M., and Straty, G. C., Thermophysical properties of ethane, from 90 to $600 \mathrm{~K}$ at pressures to $700 \mathrm{bar}$, Nat. Bur. Stand. (U.S.), Tech. Note 684, 326 pages (Aug. 1976).

[6] Goodwin, R. D., Provisional thermodynamic functions of propane, from 85 to $700 \mathrm{~K}$ at pressures to $700 \mathrm{bar}$, Nat. Bur. Stand. (U.S.) Internal Report NBSIR 77-860 (July 1977).

[7] Goodwin, R. D., On the nonanalytic equation of state for ethylene, unpublished report, this laboratory (July 1977).

[8] Goodwin, R. D., The nonanalytic equation of state for pure fluids, applied to propane, manuscript for symposium on Equations of State in Engineering and Research, 176th National Meeting of the American Chemical Society, Miami Beach, Fla., Sept. 10, 1978. 